\title{
A patient with recurrent aseptic meningitis (Mollaret's meningitis) due to herpes simplex type I infection
}

\author{
DAJ Chandrika ${ }^{1}$, KD Pathirana $^{2}$, LAAAbeywickrema $^{3}$ \\ ${ }^{1}$ Registrar in Medicine, ${ }^{2}$ Consultant Neurologist, ${ }^{3}$ Senior Registrar in Medicine, Professorial Medical Unit, \\ Teaching Hospital, Karapitiya, Galle.
}

e-mail address of the corresponding author,Dr.D.A.J.Chandrika: jananilchandrika@gmail.com

\section{Case report}

A 35 year-old woman presented with a history of fever, headache, vomiting and photophobia the day after the fullterm uncomplicated normal vaginal delivery of her second child. On examination, she was drowsy, had neck stiffness and positive Kernig's sign. Investigations showed marked neutrophil leucocytosis with high erythrocyte sedimentation rate (ESR). Cerebrospinal fluid (CSF) was markedly turbid with polymorphs of $534 / \mathrm{mm}^{3}$, lymphocytes of $3 / \mathrm{mm}^{3}$, red cells of $20 / \mathrm{mm}^{3}$, protein of $0.65 \mathrm{~g} / \mathrm{L}$ CSF glucose of $3.7 \mathrm{mmol} / \mathrm{L}$ (with a simultaneous random blood sugar of $6.8 \mathrm{mmol} / \mathrm{L}$ ). CSF culture was sterile.

She improved with intravenous cefotaxime $2 \mathrm{~g} 8$ hourly and a short course of intravenous dexamethasone $4 \mathrm{mg} 8$ hourly for three days. Fasting blood sugar, electroencephalography (EEG), contrast enhanced computed tomography (CT) of brain and renal functions were normal. She was discharged from ward after a 14 day course of antibiotics without any residual neurological disability.

Twenty four days after discharge she was readmitted with severe headache and dizziness without fever. She also had unsteadiness of gait and developed a generalized tonic clonic seizure without any mucosal injuries or incontinence in the same evening.

She did not give a history of recurrent otitis media, sinusitis or connective tissue disorders like systemic lupus erythematosus or vasculitic illnesses. She had not been on regular medication. There was no history of recurrent mouth ulcers or genital ulcers.

On examination, she was afebrile and there was neck stiffness but Kerning's sign was absent. She had a BCG vaccination scar. Optic disc margins were blurred. There were no focal neurological signs.
Full Blood Count, ESR, renal function and liver function tests were normal. C-Reactive protein (CRP) was $48 \mathrm{mg} / \mathrm{L}$. Repeat contrast enhanced CT scan showed no evidence of hydrocephalus, cysts or space occupying lesions. EEG was normal. CSF was not under pressure and its appearance too was normal. It contained polymorphs of $60 / \mathrm{mm}^{3}$, lymphocytes of $3 / \mathrm{mm}^{3}$ and red cells of $20 / \mathrm{mm}^{3}$. CSF protein was $0.65 \mathrm{mg} / \mathrm{L}$ while CSF sugar was 3.9 $\mathrm{mmol} / \mathrm{L}$. Simultaneous random blood sugar was 6.2 $\mathrm{mmol} / \mathrm{L}$. CSF culture yielded no bacterial growth.

Patient was treated with intravenous ceftazidime $2 \mathrm{~g}$ 8 hourly with a short course of intravenous dexamethasone $4 \mathrm{mg} 8$ hourly considering the possibility of recurrent pyogenic meningitis. She was symptoms free in three days. Extensive clinical search for sinusitis, middle ear infections, scalp lacerations with fracture of skull bones, congenital fistulae and other sites of infections was negative. Her chest radiograph was normal and CSF was negative for mycobacterium tuberculosis polymerase chain reaction (PCR). She had normal full blood count and ESR. Antinuclear antibody (ANA) was negative. CSF was positive for HSV-1 DNA but negative for HSV-2 on the fifth day after the second presentation. Diagnosis of recurrent aseptic (Mollaret's) meningitis was made and she was discharged on symptomatic treatment. Two weeks later she presented with another generalized seizure and treated with carbamazepine. She had been free of fits until the time of writing.

\section{Discussion}

Common causes for recurrent pyogenic meningitis include local infections like middle ear infection, chronic sinusitis, scalp infections with compound fractures of skull and CSF rhinorrhoea ${ }^{1}$. Congenital 
fistulae are another well known cause. Other causes of recurrent infective meningitis include incomplete antibiotic therapy, tuberculous meningitis and fungal infections ${ }^{1}$. In this patient, bacterial cultures were repeatedly negative and CSF did not show any fungi. PCR for mycobacterium tuberculous was negative.

Non-infective causes like systemic lupus erythematosis, Behcet's syndrome, idiosyncratic reaction to NSAIDs and leaking epindymomal cyst are also known causes of recurrent meningitis. In leaking ependymomal cysts imaging will be normal in acute stage due to leakage of fluid ${ }^{1}$.

Recurrent aseptic meningitis with CSF pleocytosis was first described by Pierre Mollaret in $1944^{2}$. Later it was established to be associated with HSV-2 $2^{1,2,3}$. Rarely HSV-1 and other herpes viruses had been implicated ${ }^{3}$. This illness is characterized by episodes of herpes meningismus, headache and fever separated by symptom free periods which can recur up to five years ${ }^{1,2}$. Each episode can last for few days to a maximum of three weeks ${ }^{2,4}$. Fifty percent of the patients may develop transient neurological symptoms ${ }^{2}$. CSF will show pleocytosis with large epithelioid cells called (Mollaret's cells). Attacks are self limiting and only symptomatic treatments is required ${ }^{2,4}$.
Presence of HSV-DNA indicates an acute infection with the virus. PCR for HSV-DNA in CSF has a sensitivity of more than $95 \%$ and specificity of more than $95 \%{ }^{1}$. Antiviral therapy with acyclovir is indicated only in severe cases ${ }^{2}$. Place of longterm prophylaxis antiviral therapy is not known ${ }^{2}$. This case is unusual in that the infection is due to HSV-1 and the patient had seizures in addition to recurrent meningitis.

\section{References}

1. Roberta L DeBiasi, Marylou V Solbrig, Kenneth L Tyler. Infections of the Nervous System in: Walter G Badley, Robert B Daroff, Gerald M Fanideel, Joseph Jankonn (Editors) Neurology in Clinical Practice. $4^{\text {th }}$ Edition, Elsevier 2004; 2: 1515-20.

2. Beloo Mirakhur, Marc McKenna, Recurrent Herpes Simplex Type 2 virus (Mollaret) Meningitis: Journal of the American Board of Family Practice 2004; 17: 303-5.

3. Laura Kupila, Raija Vainionpaa, Reijo J Marttila, Pirkko Kotilainen, Recurrent Lymphocytic Meningitis. Archives of Neurology 2004; 61: 1553.

4. Christopher J Boes, David J Capobianco, F Michael Cutrer, David W Dodick, Eric J Eross, Jerry W Swanson. Headache and other cranio-facial pain. In: Walter G Badley, Robert B Daroff, Gerald M Fanideel, Joseph Jankonn (Editors). Neurology in Clinical Practice. $4^{\text {th }}$ Edition Elsevier 2004; 2: 2062 